\title{
Research on Line Modeling in Painting
}

\author{
Yawei Lian \\ Nanjing Institute of Tourism \& Hospitality \\ Nanjing, China \\ e-mail: 8.lyw@163.com
}

\author{
Hanchun Zhao \\ Anhui Normal University \\ Wuhu, China \\ e-mail: 2240707022@qq.com
}

\begin{abstract}
As a natural instinct for the modeling of human beings, line can be observed everywhere in our daily life. As a result of compressing a surface under a specific perspective, line lies between a spot and a surface, represents a spot in movement, and creates a surface during movement. Existing in many forms, line can not only maintain inspiration for painters, but also create perfect works in connection with other elements. Attention has been paid to the formation and the sense of form of lines. In addition, line is also full of emotion and rhythm. By making good use of the rhythm of line, artists can express their emotions very well. Chinese and Western artists not only differ in their thinking styles and aesthetic habits, but also vary in the artistic style of applying lines. A clear understanding of this will considerably promote our comprehension of art.
\end{abstract}

\section{Keywords_line; forms of line; rhythm; difference}

\section{LINE MODELING IS A NATURAL INSTINCT}

During their artistic activities, primitive human beings instinctively used line as their painting device. The extensive application of line can be noticed from primitive paintings on colored pottery, mural paintings, cliff paintings and Lascaus Grotto Murals (fig.1) discovered in modern times. The line-sketch works of some artists in contemporary times (e.g. Henry Moore and Sculptor W Otter), or the lines sketched for their works seem to have been applied freely without much hesitation, as if the line forms were taken for granted, so that the pictures appear quite relaxed and expression with lines appears inborn.

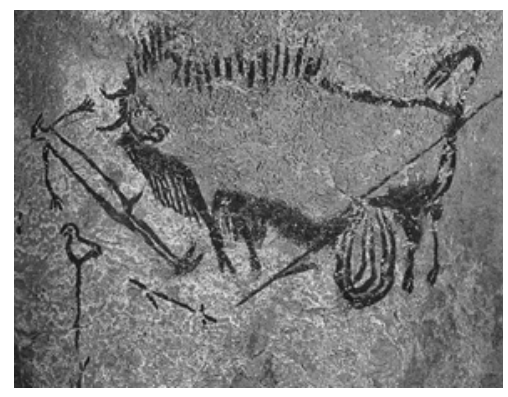

Fig.1. Lascaus Grotto Murals

\section{LINES IN DAILY LIFE}

As an old Chinese saying goes, "human beings and the mother nature can transform each other”. At any moment, we can transform our feelings into visual forms by observing our life and perceiving the mother nature, and conclude the law and methods for aesthetic expression. Our works originate from but exceed the nature. We may turn a blind eye to lines of objects every day, because we overlook their intrinsic structure and rhythm. For example, although water surface is a visual feeling, the parallel lines of "ripples in spring" can be sketched in a plain way. This is exactly what we can refine from our daily life according to our feelings. Emotionally-perceived lines exist in every corner of our daily life.

\section{THE GENERATION OF LINE}

Not existing in itself, line is noting but a special state of existence of objects. Line results from the extreme perspective-based shrinkage of the surface of an object parallel with the sight, except the invisible surface blocked by the object itself. Often manifested as the edge of a shape, this line is called "contour line". Within the contour line, the line which can be perceived visually at the place for the transition and connection of surfaces of object is exactly structure line, or inner contour line. We can apply structure line to determine the volume and spatial location of an object. This kind of sketch is characterized by: using line as a way of expression; applying no light and shade; having no light and shadow change; emphasizing the structural feature of object; aiming to express the self-structure of object; using the ways of observation, reasoning and perspective at the same time; not only attaching importance to an intuitive way.

\section{FORMS OF LINE}

As a modeling element, line would undergo a change in its depth, density, thickness, curvature, length, visibility, rigidness, tempo and weight. In order to mold images, Chinese artists have conducted intensive research on lines. According to the relevant record in Enlightenment of Paining by Zou Dezhong in the Ming Dynasty, line includes 18 sketching techniques: e.g. string sketching, iron-wire sketching, gossamer sketching, smooth sketching, nail-head and rat-tail sketching, ripple sketching, orchid-leave sketching, sketching of reduced strokes and mixed sketching. By using different techniques, we can sketch lines with different feelings in thickness, curvature, roundness, rigidness, hair-handiness, weight, visibility, complexity, smoothness and regularity. We can compose various line-modeled forms on pictures by using the gradual change, repetition, arrangement, combination, intersection, segmentation, echo and contrast of these lines. The above-mentioned lines not only represent the high-level generalization of painters in the past dynasties, but also their rich experience in lines and the technique of 
sketching. They have set up a complete set of techniques of applying the brush for lines. These special lines are very suitable for expressing painters' emotions and their feelings of objects.

\section{THE EMOTION AND RHYTHM OF LINE}

Line has a very strong force of expression. The rigidness, pause, transition, smoothness and other changes of line (fig.2) can express the texture and rich connotation of objects. We can achieve our goals by using different lines according to characteristics of objects. For example, rigid, thick and heavy lines had better be used to express plain and solid objects, and curvy and delicate lines had better be used to depict smooth and exquisite objects. In addition, the expressive force of a single line is very leisurely. A group of lines can constitute various feelings very well. Various contrasts can come into being among different lines, thus bringing about the rhythm of curvature/straightness and square/roundness. The rhythm of fineness, coarseness, smoothness and intermittence can also bring about such aesthetic feelings as roundness, primitiveness and uprightness. In order to express the structure of objects and the transition of surfaces, we should use definite, positive and solid lines. As for other parts, we should use weakened and dotted lines. As for physical spaces, we should use solid, thick and heavy lines and dotted, fine and light lines to express the front and the rear of the objects respectively.

Thick straight line: With a strong and vigorous expression, thick straight line can give a sense of shock and impact. Fine straight line: With an emotional expression of tenderness and reliance, fine straight line gives a sense of extension and comfort. Thick curved line: With a calm and slow expression, thick curved line gives a sense of depression and stillness. Fine curved line: With an emotional expression of lightness and softness, fine curved line gives a sense of elegance and mystery. Free line: With a changeable and relaxed expression, free line gives a sense of relaxation and unrestraint. Standard line: With an expression of quietness and steadiness, standard line gives a sense of conservation and preciseness. Independent curved line: With a lonely and independent expression, independent curved line gives a sense of stillness. Continuous curved line: With a dynamic expression, continuous curved line gives a sense of life and motion. For example, in Picasso's Girl Before a Mirror (fig.3), Picasso used thick and wild lines to draw the human body of the girl, used thick lines to draw her contour, back and waist, and used fine lines to draw her neck. With the change between thickness and fineness, the girl's contour drawn by Picasso is more beautiful and charming, and the shape of the girl is more vivid. The straight lines in Concert on Broadway by Mondrian are very orderly, thus giving a sense of serenity. Therefore, the change of lines can make objects on pictures more vivid and prominent and the pictures more expressive.

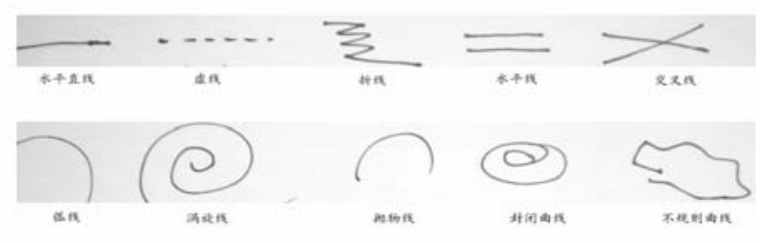

Fig.2. Horizontal straight line; Dotted line; Folded line; Parallel lines; Crossed lines; Arc line; Vortex line; Parabola; Closed curved line; Irregular curved line.

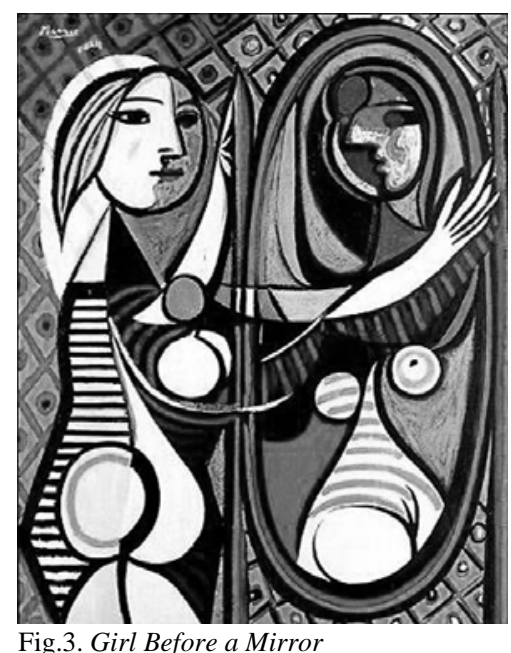

\section{THE DIFFERENCE BETWEEN AND THE CHARACTERISTICS OF CHINESE AND WESTERN APPLICATION OF LINES}

Westerners attach more importance to objective laws and "realistic painting". Therefore, they regard lines as a part of objects and emphasize exogenous action. In contrast, Chinese attach more importance to the expression of subjective emotions and pursuit of images. Therefore, Chinese pursue artistic conception outside pictures rather than apparent similarity. The difference between Chinese and Western lines is actually that between Chinese freehand outlook and Western realistic outlook.

As a basic modeling element, line in Western paintings pursues a sense of expression, a feeling of quantity and a modeling effect. In contrast, in Chinese paintings, line lays more emphasis on expressing the painter's thoughts and emotions, and conveying the painter's subjective feelings and ideas. For example, in Admonitions Scroll and Ode to the Goddess of Luo by Gu Kaizhi, lines are used like a thread of silkworm and a floating cloud in the sky. In Black Grape by $\mathrm{Xu}$ Wei, crooked lines are used as freely as possible. However, these lines are applied very skillfully and effectively. Lines are used to draw grape branches and leaves in different forms, so as to express sentiments. The lines used by Chen Laolian are very connotative. Line sketches of traditional Chinese paintings have gradually become a form with an independent value of aesthetics, and many genres have come into being at the same time. Traditional Chinese paintings which use line as their modeling means have a unique value of aesthetics. By learning line sketches of traditional Chinese paintings, we can promote the expressive force of lines in modeling. 
Lines in Western paintings have the function of defining shapes. Attached to shapes, lines are not a major modeling element. Line lays foundation for the existence of shapes or structures. For example, some contour lines serve to resurrect the structure of objects. Western paintings attach importance to shape modeling by relying on colors and block-surfaces. The major languages applied for modeling are still shapes and colors. It does not matter much even if there is no line. Although some Western paintings also model shapes with lines, the main function of these lines is to define the contour or structure.

Excellent paintings based on line modeling have ever emerged in Greek, Egypt, Babylon, Mayan Civilization, and India. Many masters of line modeling have also emerged in Europe. For example, Ingres, Durer, Picasso, and Mattisse have all created masterpieces with lines. Miro's lines are full of naive and unsophisticated beauty. Mattisse's lines are full of pure and general beauty. Mondrian pursued the straight-line segmentation in solid geometric spaces, uttering the same emotion with vertical and horizontal lines, and creating a three-dimensional world of lines. In the framework of straight lines, he displayed the rationalism of the Science Age and pursued the beauty of orderliness and rationality. Its influence can be discovered in industrial, architectural and other designs.

These Chinese and foreign masters have provided us with abundant artistic nutrients. We should learn and imitate their essence, continuously deepen our understanding of lines, and grasp the law and skills of line application, so that line can become a medium for us to express our emotions.

\section{REFERENCES}

[1] Yu Aijun, Book on Sketching (M), Liaoning Province: Liaoning Fine Arts Publishing House, 2005

[2] Tang Dinghua, Fundamental Sketching (M), Beijing: People's Fine Arts Publishing House, 2011 